\title{
La Bioeconomía en el Mundo Moderno: una revisión de literatura desde los 5 continentes*
}

\author{
Bioeconomics in the modern world: a literature review from the 5 continents. \\ A bioeconomia no mundo moderno: uma revisao da literatura desde os cinco continentes
}

DOI: https://doi.org/10.21803/pensam.v17i21-1.278

\author{
Ana Cristina Zúñiga Zapata \\ https://orcid.org/0000-0001-6503-412x
}

Eduardo Arturo Cabezas Aguilar https://orcid.org/0000-0002-0544-4650

Elkin Olaguer Pérez Sánchez https://orcid.org/0000-0002-0544-4650

\section{¿Cómo citar este artículo?}

Zuñiga, A., Cabezas, E. \& Pérez, E. (2018). La Bioeconomía en el Mundo Moderno: una revisión de literatura desde los 5 continentes. Pensamiento Americano, 11(22), 230-246. DOI: https://doi.org/10.21803/pensam.v17i21-1.278

\begin{abstract}
Resumen
El artículo hace un recorrido por los países del mundo que han documentado y puesto a circular información acerca de la Bioeconomía y han hecho definiciones explicitas de políticas o estrategias, y además han establecido lineamientos para su desarrollo. Se entiende la Bioeconomía como la gestión eficiente y sostenible de la biodiversidad para generar nuevos productos, procesos y servicios con valor agregado, evitando el uso de los recursos naturales no renovables con el fin de promover la innovación tecnológica, desarrollar empleos cualificados y mejorar la calidad de vida de las comunidades en cada una de las localidades y de los países en general. Para este recorrido se hizo una revisión de literatura en bases de datos. Como resultado se presenta a la comunidad académica un panorama de la orientación que se está dando a la bioeconomía en los diferentes países del mundo.
\end{abstract}

Palabras Claves: bioeconomía, biomasa y sostenibilidad, biodiversidad, desarrollo sostenible, políticas.

\begin{abstract}
The article takes a tour of the countries of the world that have documented and circulated information about the Bioeconomy and made explicit definitions of policies or strategies, and established guidelines for its development. Bioeconomy is understood as the efficient and sustainable management of biodiversity to generate new products, processes and services with added value, avoiding the use of non-renewable natural resources in order to promote technological innovation, develop qualified jobs and improve quality of life of the communities in each of the localities and the countries in general. For this tour, a review of literature in databases was made. As a result, an overview of the orientation that is being given to the bioeconomy in the different countries of the world is presented to the academic community.
\end{abstract}

Key words: bioeconomy, biomass and sustainability, biodiversity, sustainable development, policies.

* Articulo resultado de investigación del proyecto de tesis doctoral denominado: Bioeconomía y desarrollo socio económico en Colombia: retos y oportunidades para un país megadiverso. 


\section{Resumo}

Este articulo elabora um recorrido pelos países do mundo que documentaram e circularam informação sobre a Bioeconomia e definiram explicitamente políticas ou estratégias, y estabeleceram lineamentos para seu desenvolvimento. Entende-se a Bioeconomía como a gestão eficiente e sustentável da biodiversidade para gerar novos produtos, processos e serviços com valor agregado, evitando o uso dos recursos naturais não-renováveis com a finalidade de promover a inovação tecnológica, desenvolver empregos qualificados e melhorar a qualidade de vidas das comunidades em cada uma das localidades, e dos países em geral. Para isso, houve uma revisão da literatura nas bases de dados. Como resultado, apresenta-se à comunidade acadêmica um panorama da orientação que se está dando à Bioeconomia nos diferentes países do mundo.

Palavras chave: bioeconomia, biomassa e sustentabilidade, biodiversidade, desenvolvimento sustentável, políticas

\section{Perfil}

Dirección De Investigación, Corporación Universitaria

Adventista,investigacion@unac.edu.co

Ana Cristina Zúñiga Zapata Magister en Gestión de Ciencia

tecnología e innovación.

\section{Perfil}

Coordinador de la Maestría en Administración- MBA, Coordinador Maestría en Innovación, Universidad de Medellín, Medellín, Colombia, eoperez@udem. edu.co.

\section{Perfil}

Magister en Administración, magister en Tecnologías de la Información Aplicadas a la Educación. especialista en docencia universitaria. Universidad de Medell.n, edu_artu5@hotmail.com. investigacion@unac.edu.co

\section{Eduardo Arturo Cabezas Aguilar}

Ph.D en administración.

Elkin Olaguer Pérez Sánchez

Ph.D en Administración 


\section{Introducción}

El concepto de cuarta Revolución industrial, se viene discutiendo en los círculos académicos acerca de la importancia que tiene para el mundo el surgimiento de las nuevas tecnologías que ayuden a satisfacer las necesidades básicas de la comunidad en general y al cumplimiento de los objetivos de Desarrollo Sostenibles (ODS).

La bioeconomía tiene como objetivo generar respuestas a los desafíos y retos asociados a las problemáticas mundiales, especialmente lo relacionado con la mitigación del cambio climático, es claro que los problemas comienzan en las regiones y se extienden al alcance global. Es difícil cuantificar lo que va a ser más difícil al enfrentar estos desafíos, Sin embargo, una de las visiones para la bioeconomía, es la necesidad de tener un panorama claro de cuáles son las fortalezas y oportunidades que cada una de las regiones del mundo ofrece a partir de su ubicación geoestratégica y las características particulares de sus ecosistemas.

Cada día se hace más evidente que la bioeconomía se ha transformado en un asunto transcendental para la elaboración de políticas de investigación e innovación, esencialmente para países industrializados (Meyer, 2017). La bioeconomía en términos político y económico plantea la sustitución de recursos fósiles por recursos biológicos para contrarrestar el problema del cambio climático y detener el empobrecimiento de los recursos naturales (Asada \& Stern, 2018), aunque se encuentra perjudicada por diferentes factores como la biomasa disponible y el desarrollo o la obtención de tecnologías de biorrefinería (Sillanpää \& Ncibi, 2017).

Los gobiernos junto a su administración participativa y adaptiva necesitan garantizar que todos los actores de cambio estén inmersos como un asunto de justicia básica en los diferentes desarrollos de transición de la bioeconomía, es decir la implementación de los valores ciudadanos, los intereses, los conocimientos técnicos y los derechos ambientales (Mustalahti, 2017).

Por otro lado, Scarlat, Dallemand, Monforti-Ferrario y Nita (2015) estiman que el mercado de la bioeconomía estaría rondando los 2.400 millones de euros, en los próximos años, en donde los sectores de los alimentos y bebidas, agricultura, pesca, acuicultura, productos agroindustriales, madera y silvicultura mueven alrededor de 2 mil millones de toneladas y se emplean 22 millones de capital humano, además gracias a la bioeconomía se están formando nuevos sectores como los biomateriales y la química verde. Otros modelos bioeconómicos han direccionado su desarrollo lineal de refinería petroquímica al de una biorrefinería (Aguilar, Wohlgemuth \& Twardowski, 2018).

Cada vez se incrementan más los países que desean desarrollar sus estrategias de bioeconomía buscando empezar una mejor credibilidad y confianza en la utilización eficiente de los recursos biológicos renovables, con las finalidades de cumplir con los diferentes retos que otorga la sostenibilidad (Biber-Freudenberger, Kumar, Bruckner \& Börner, 2018).

Estudios e investigaciones elaboradas por German Bioeconomy Council (2015) nos indican que existen, alrededor de 45 países que han documentado y puesto a circular información acerca de la Bioeconomía y la definición explicita de políticas o estrategias, y además han establecido lineamientos para su desarrollo. Al hacer un análisis encontramos los países que en los cinco continentes tienen explicitas las políticas y estrategias: América (8), Europa (16), Asia (9) Oceanía (2), África (10).

El objetivo central del artículo es hacer un re-

Pensamiento Americano Vol. 11 (22) · 2018 • Julio-Diciembre · Corporación Universitaria Americana • Barranquilla, Colombia • ISSN: $2027-2448$. 
corrido por los países del mundo que han documentado y puesto a circular información acerca de la Bioeconomía y han hecho definiciones explicita de políticas o estrategias, y además han establecido lineamientos para su desarrollo, para este recorrido se hizo una revisión de literatura en bases de datos, que permitió identificar como cada país dentro de sus políticas o estrategias consideran diferentes lineamientos, como se muestra en la Figura 1, orientados hacia la Biotecnología, Economía Verde, Economía Circular, Bioenergía, Economía de base biológica, Economía Azul, Investigación, Innovación y Desarrollo, Desarrollo Holístico, Ciencias de la vida, Agrociencia y Tecnología, Desarrollo Regional, Agricultura, Química Verde (Dieckhoff, El-Cichakli \& Patermann, 2015).

Los gobiernos o responsables en direccionar las políticas en términos de bioeconomía encuentran un futuro complicado y diverso, es difícil medir cual es o será es desafío más complicado, ya que los inconvenientes parten desde las regiones y se amplifican globalmente (Philp, 2018). Por su parte Goven y Pavone (2015) argumentan que la bioeconomía tiene que comprenderse como un proyecto político y no estrictamente como tecnocientífico y económico, por ejemplo, uno de los enfoques de la bioeconomía, es la fabricación racional de biorrefinería en pequeña y mediana escala, pero este enfoque lucha con la realidad actual de las economías masivas de petroquímicos y combustibles fósiles que en muchas ocasiones están beneficiadas de subsidios (Philp, 2018). Lo que se espera es que a mediano plazo el desarrollo compuesto por todas las áreas o sectores relacionados a la bioeconomía ayude a solucionar los problemas de seguridad alimentaria mundial, optimizar la nutrición y la salud pública, forjar que los procesos industriales sean mucho más limpios y eficaces y favorecer en gran magnitud al impulso de mitigar

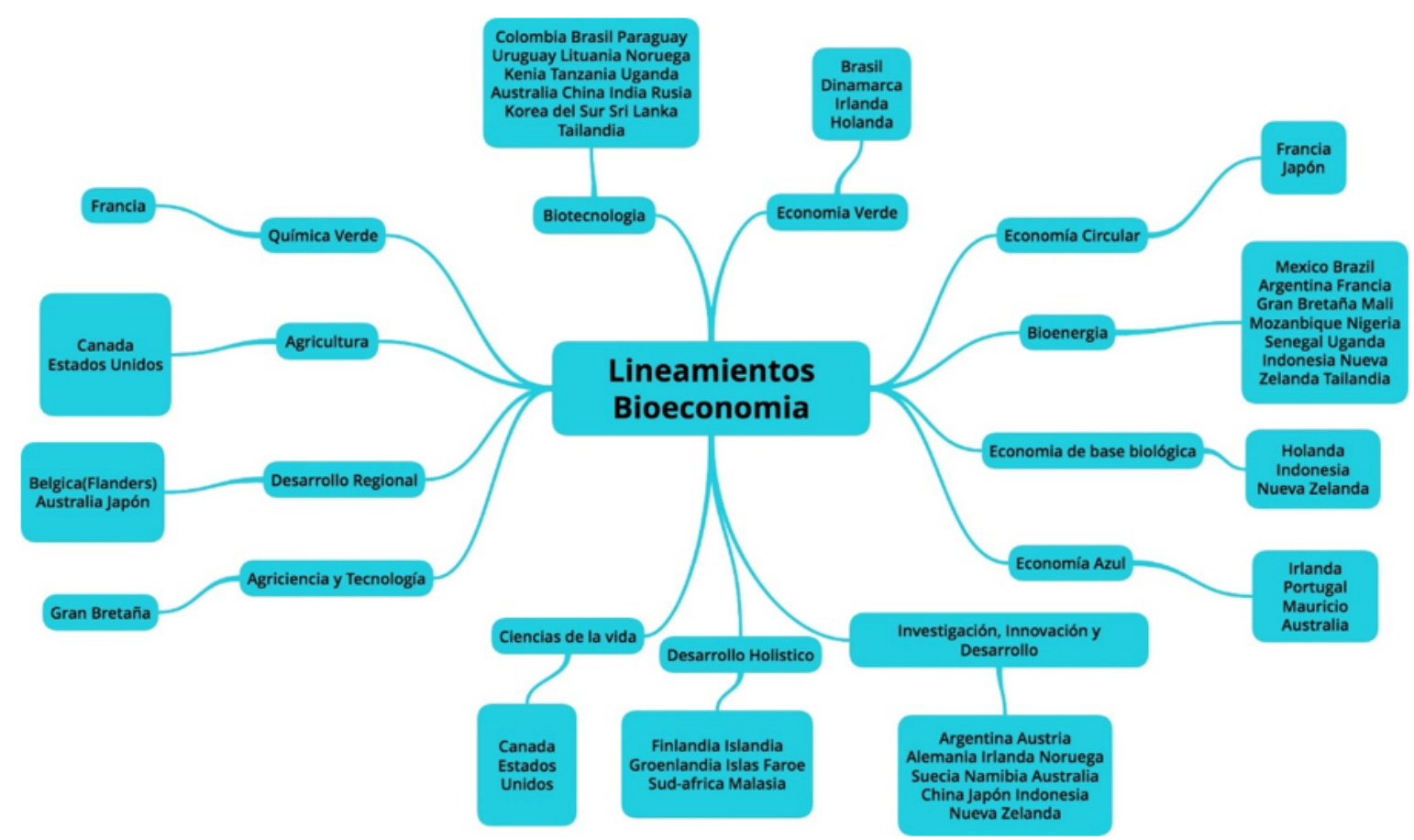

Figura 1. Lineamientos de la Bioeconomía en el mundo por paises.

Fuente: Elaboración propia con base en la revisión de literatura. 
el cambio climático (Lokko et al., 2018).

\section{METODOLOGIA}

En el desarrollo del artículo se utiliza una revisión de literatura, para lo cual los datos utilizados se tomaron de publicaciones ISI web of Science y Scopus, publicadas entre los años 2013 y 2018. Para la estrategia de búsqueda de los documentos se tuvieron en cuenta como ecuaciones de búsqueda "bioeconomía", "Bioeconomía + estrategias + políticas en el mundo" Así se obtuvieron 72 artículos. Estos artículos fueron analizados sobre la base metodológica de la Revisión de Literatura (Álvarez, Amorós \& Urbano, 2014). A partir de este análisis se seleccionaron finalmente un total de 32 artículos, que son los que se presentan en esta Revisión de Literatura.

La Figura 1, muestra las etapas utilizadas para la escritura del artículo. Se explica cómo fue desarrollada una metodología que integró las técnicas de RL con la de análisis de contenido, con base en el estudio de la información de los campos bibliográficos de artículos científicos y estructurada en bases de datos. En la primera etapa se hizo una identificación de las fuentes de donde se quería obtener la información, para comprobar su relevancia en el campo de estudio. La segunda etapa permitió hacer un análisis bibliométrico a partir del contenido de los documentos científicos, y en la tercera etapa se hizo el análisis definitivo de los artículos.

\section{RESULTADOS}

Países de América con políticas y estrategias de Bioeconomía explicitas.

Se encontraron en América 8 países que han documentado y puesto a circular información acerca de la Bioeconomía y la definición explicita de políticas o estrategias, y además han establecido lineamientos para su desarrollo: 3 en Norteamérica (Canadá, Estados Unidos y México) y 5 en Sudamérica (Colom-

\section{Etapa I}

Revisión de información

Etapa II

Análisis de la información

Etapa III

Análisis de contenidos $\checkmark \quad$ Identificación de fuentes de información (ISI web of Science)

$\checkmark \quad$ Identificación de palabras clave

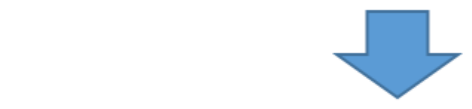

Definición de criterios de búsqueda

Búsqueda y procesamiento de la información

$\checkmark \quad$ Análisis de los artículos

Figura 2. Etapas utilizadas para la revisión de literatura.

Fuente: Elaboración propia
Selección de artículos relevantes de acuerdo con el objeto a investigar

$\checkmark$ Análisis de los artículos seleccionados 
bia, Brasil Argentina, Paraguay y Uruguay), como se muestra en la Tabla 1.

\begin{tabular}{|c|c|}
\hline \multicolumn{2}{|l|}{ Lineamientos de los paises de América } \\
\hline Orientación de la estrategia de Bioeconomía & País \\
\hline $\begin{array}{l}\text { Ciencias de la vida (Biomedicina) Agricultura (áreas } \\
\text { múltiples) }\end{array}$ & Canadá \\
\hline $\begin{array}{l}\text { Ciencias de la vida (Biomedicina) Agricultura (áreas } \\
\text { múltiples) }\end{array}$ & $\begin{array}{l}\text { Estados Unidos de } \\
\text { Norteamérica }\end{array}$ \\
\hline Bioenergía & México \\
\hline Biotecnología & Colombia \\
\hline \multicolumn{2}{|l|}{ Bioenergía } \\
\hline Biotecnología & Brasil \\
\hline \multicolumn{2}{|l|}{ Economía verde } \\
\hline Bioenergía & Argentina \\
\hline Investigación e Innovación & Argentina \\
\hline Biotecnología & Paraguay \\
\hline Biotecnología & Uruguay \\
\hline
\end{tabular}

Estados Unidos y Canadá establecen que la biomedicina es una promotora de la bioeconomía, como también es un sector estratégico para su desarrollo, Estados Unidos es el único país en el Norte de América que ha propuesto una estrategia bioeconómicos orientada a ser líderes en tecnología y al crecimiento del mercado de las bioindustria, ambos países al ser beneficiados geográficamente por muchos recursos, son los que fomentan económicamente actividades de emprendimiento e innovación en el sector de producción primaria (Dieckhoff et al., 2015).

Algunos sectores económicos de Canadá como: el transporte y energía, productos farmacéuticos, alimentos y agroalimentos, silvicultura, materiales y fabricación, nutraceúticos, gestión de desechos y una vasta variedad de recursos de consumo estarían afectados por la bioeconomía, y gracias a su intervención el país podría lograr una renovación económica en estas y otras áreas en donde bienes naturales no han podido proporcionar de un empleo sostenible, uno de los sectores primordiales que Canadá espera que la bioeconomía respalde y contribuya en su desarrollo es el sector forestal (Duchesne \&v Wetzel, 2003).
Aunque Canadá no mantenga una estrategia específica de Bioeconomía maneja 4 sectores estratégicos que son BioSalud, Bioenergía, Bioindustrias y Biotecnología agrícola (Biotalent Canadá, 2013) como se muestran en la Figura 2.

Según el Departamento de Agricultura de los Estados Unidos (USDA, 2015) la bioeconomía genera en Estados Unidos grandes oportunidades de crecimiento como por ejemplo en el impacto económico: 369 billones de dólares, además en 2013 se generaron 4 millones de

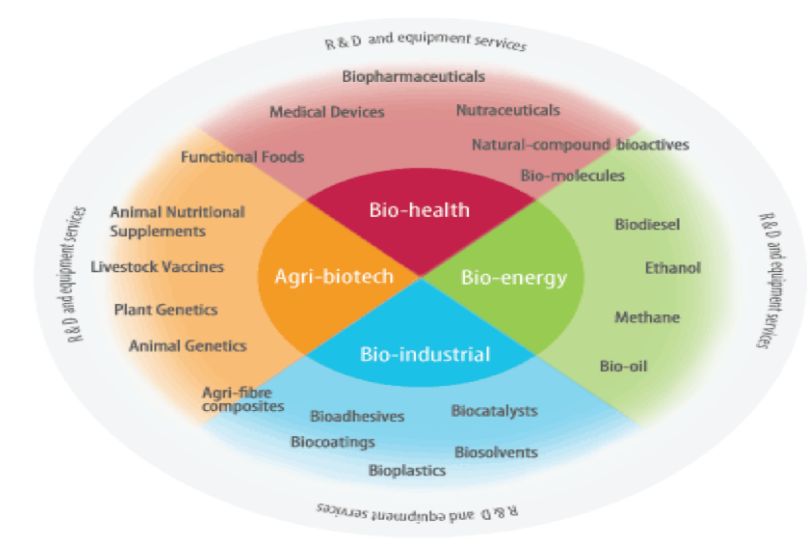

Figura 3. Sectores de la bioeconomía y productos.

Fuente: (Biotalent Canadá, 2013)

empleos y se logró la venta de 126 billones de dólares en productos relacionados a la bioeconomía, en el ámbito social 300 millones de galones de petróleo fueron reemplazados por productos biobasados, se retiraron 200.000 vehículos que emitían gases de efecto invernadero y se certificaron 2.250 productos biobasados en el mercado (Golden, Handfield, Daystar \& McConnell, 2015).

Además, el reporte del German Bioeconomy Council (2015) nos dice que los grandes retos bioeconómicos de Estados Unidos están enfocados según su lineamiento en la Biomedicina y la Agricultura siguiendo el Bioeconomy Blueprint 2012, el mismo que se con- 
centra particularmente en las biotecnologías, como las ingenierías genéticas, microbios y plantas vivas, los procesos del ADN, la utilización de microorganismos o fermentaciones industriales, siendo su propósito principal la disminución del uso de hidrocarburos para la producción energía en el país (Henry, Pahun \& Trigo, 2014).

En México la bioeconomía está alineada con la Bioenergía (German Bioeconomy Council, 2015), el desarrollo de la Bioenergía es muy importante porque apoya a la seguridad alimentaria y energética, y al mismo tiempo disminuye la polución ambiental, sin embargo, uno de los propósitos principales de la estrategia es promulgar el desarrollo rural perfeccionando la producción agrícola y endureciendo la agroindustria mexicana.

La estrategia en Bioenergía establece normas burocráticas que promueven un ambiente adecuado para una gran inversión, crean oportunidades laborales, vigilan las existencias y el uso de sembríos de bioenergía, crean principios de sostenibilidad y salvaguardan la biodiversidad del país (Dieckhoff et al., 2015)

En América Central y del Sur el desarrollo de la bioeconomía está caracterizado por las estrategias políticas que promueven primordialmente la capitalización de la enorme cantidad de recursos naturales de estos países (Dieckhoff et al., 2015). Por ejemplo América Central debe proteger y mantener su gran potencial de abastecimiento de recursos naturales como lo son el agua y la biodiversidad (Vega \& Madrigal, 2017). Según CEPAL en el 2017, nos dice que en América Latina existe un gran potencial para desarrollar la Bioeconomía, la misma que permitiría una gran alternativa para la transformación productiva y la incorporación de valor en los sectores agrícolas y agroindustriales, fundamentalmente en los medios rurales.
German Bioeconomy Council (2015) tiene en cuenta a cinco países latinoamericanos (Colombia, Brasil, Argentina, Paraguay y Uruguay) que, aunque no tienen definidas sus estrategias o políticas, si tienen presente la Bioeconomía para su desarrollo.

Brasil es un claro ejemplo en el desarrollo de la bioeconomía avanzada, está sustentada principalmente en el conocimiento y entendimiento en la fabricación de etanol (Sasson \& Malpica, 2017), el apoyo político durante décadas hizo que la producción de bioetanol sea muy competitiva (Lewandowski, 2015), de igual manera la producción de caña de azúcar de tipo Geneticamente Modificada (GM) que es resistente a plagas, además el país se interesa en el uso cepas de levadura y diferentes tipos de microorganismos, reciclaje de desperdicios, como por ejemplo el bagazo de la caña de azúcar los incluyen en el ciclo de energía. Brasil mira estos desarrollos y avances como un gran sentido en vista hacia una economía verde o bioeconomía (Sasson \& Malpica, 2017).

Uno de los principales desafíos que tiene América Latina se encuentra en la implementación de las actuales cadenas de valor en relación a la bioeconomía, en la cual los recursos tecnológicos se localizan en un nivel muy bajo y el sector económico está aún en desarrollo, como por ejemplo en el sector de biocombustibles y bioproductos (Sasson \& Malpica, 2017).

La región Latino Americana y el Caribe se encuentran muy bien ubicadas para aportar y aprovecharse de la Bioeconomía emergente (Trigo et al., 2013), además la región constituye un gran capital de biodiversidad, este desafío de precautelar, cuidar y mantener los bienes ecosistémicos demandan soluciones locales y regionales (Sasson \& Malpica, 2017). Aquí es importante mencionar un grupo de países

Pensamiento Americano Vol. 11 (22) • 2018 • Julio-Diciembre · Corporación Universitaria Americana · Barranquilla, Colombia • ISSN: $2027-2448$. 
megadiversos, entre los que se encuentran México, Venezuela, Colombia, Ecuador, Perú, Bolivia y Brasil, pero también se debe tomar en cuenta a Guatemala, Costa Rica y Panamá quienes cuentan con Índices Nacionales de Biodiversidad. Toda esta región es reconocida por su magnitud de recursos naturales (tierra, agua y biodiversidad) y son los principales factores que determinan un incremento en valores estratégicos para un universo biológico (Trigo et al., 2013).

La bioeconomía en la región Latinoamericana y del Caribe conforma dos objetivos específicos: 1) En el entorno mundial, cumplen con un título esencial en el aporte a los balances globales de alimentos, fibra y energía, además regenera la sostenibilidad ambiental y 2) En el entorno regional, con la implementación de la bioeconomía se crean nuevas oportunidades de crecimiento y desarrollo sin brechas, es decir mediante el aprovechamiento de la producción agrícola y de la biomasa perfeccionada (Trigo et al., 2013).

\section{Países de Europa con políticas y estrategias de Bioeconomía explicitas.}

En este año, se cumplen 5 años de existencia de la estrategia de bioeconomía de la Unión Europea, la misma que ha gozado de triunfos en varias áreas, como por ejemplo muchos estados miembros y regiones ya desarrollan sus propias hojas de ruta y establecen estrategias de especialización inteligente, es decir establecieron que la bioeconomía va a desempeñar un presente y futuro más sostenible para Europa y sus habitantes (Dupont-Inglis \& Borg, 2018).

La Comisión Europea con el propósito de enmarcar un inicio de la bioeconomía, ha propuesto varias estrategias para edificar una economía sostenible relacionada a la biodiversidad como una gran oportunidad para entrar en discusión a varios problemas como la seguridad alimentaria, dependencia de recursos fósiles, agotamiento de recursos naturales y el enorme cambio climático, resaltando la utilización razonable y sostenible de los recursos naturales y el medio ambiente (Scarlat et al., 2015). Así mismo, reconoce peligros potenciales que no se deben pasar por alto, como son: 1) competitividad entre el abastecimiento de alimentos y la fabricación de biomasa, 2) reindustrialización y monopolios de la producción agroalimentaria, 3) explotación masiva de recursos naturales y disminución de biodiversidad y 4) perjuicio en la creencia del consumidor (Imbert, Ladu, Morone \& Quitzow, 2017).

El reporte del German Bioeconomy Council (2015) menciona que solamente 16 países europeos (Alemania, Austria, Bélgica (Flanders), Dinamarca, Francia, Reino Unido, Finlandia, Irlanda, Lituania, Holanda, Noruega, Portugal, Suecia, Islandia, Groenlandia e Islas Feroe) tienen presente a la bioeconomía como estrategia o política nacional, con su respectivo lineamiento, como se muestra en la Tabla 2. La bioeconomía en la Unión Europea tiene sus orígenes en las necesidades y en la casualidad (Patermann \& Aguilar, 2018).

En el año 2002, la Comisión Europea confirmo que la biotecnología y las ciencias de la vida se encuentran dentro de las tecnologías más competentes y de gran alcance para aportar al logro de los objetivos de la Agenda de Lisboa, tres años después en el 2005 se realizó una ponencia internacional sobre la bioeconomía fundamentada en el conocimiento, en el año 2007 continuó la ponencia pero esta vez marcando un hito importante donde se presentaron los panoramas sobre la bioeconomía europea para los siguientes 20 años. Gracias a estas conferencias se le otorgó a la bioeconomía gran importancia en los círculos políticos europeos (McCormick \& Kautto, 2013). 
Tabla 2

Lineamientos de los países europeos

\begin{tabular}{ll}
\hline \multicolumn{1}{c}{ Orientación de la estrategia en Bioeconomía } & \multicolumn{1}{c}{ País } \\
\hline Investigación e Innovación & Austria \\
Desarrollo regional Bioeconómico & Bélgica (Flanders) \\
Economía verde & Dinamarca \\
Bioenergía, Química verde, Economía circular & Francia \\
I+ D sobre seguridad alimentaria, agricultura & \\
sostenible, nutrición saludable, procesos & Alemania \\
industriales, bioenergía & \\
Bioenergía, agro-ciencia y tecnología & Gran Bretaña \\
Desarrollo holístico de la bioeconomía & Finlandia \\
Economía azul & \\
Economía verde & Irlanda \\
Investigación e innovación & \\
Biotecnología & Lituania \\
Economía verde & Holanda \\
Economía de base biológica & \\
Investigación y Desarrollo & Noruega \\
Biotecnología & Portugal \\
Economía azul & Suecia \\
Investigación e Innovación & Islandia, Groenlandia, \\
Desarrollo holístico de la bioeconomía & \\
\hline
\end{tabular}

Fuente: (Dieckhoff et al., 2015; Fund et al., 2015).

Para el año 2012, la Unión Europea exhibió su estrategia inicial de bioeconomía orientada en el perfeccionamiento de nuevos procesos tecnológicos, impulso a los mercados y a la competencia y el estímulo para una cercana cooperación entre los mandatarios políticos y las partes interesadas (Fund, et al., 2015). En el mismo año la Comisión Europea fundamentándose en las conclusiones de los informes y consultas de los interesados, difundió un documento que combina estrategias y planes de acción llamado "Innovando para un crecimiento sostenible: una bioeconomía para Europa", que tiene como metas principales brindar las guías necesarias para las agendas de innovación e investigación en las áreas de bioeconomía, el apoyo a un ambiente político más próspero y buscar las vías para una comunidad europea más innovadora, competitiva y eficaz en el uso de los recursos (De Besi \& McCormick, 2015). La Comisión Europea puntualiza que la bioeconomía es un elemento estratégico para el crecimiento y desarrollo inteligente y ecológico; de allí que por ejemplo la bioeconomía europea ocupa una dimensión en el mercado de más de 2 billones de euros y suministra de 22 millones de empleos en sectores como la bioenergía, agricultura, alimentos, silvicultura y productos químicos, que significan el 9\% del poder laboral total de la Unión Europea (McCormick \& Kautto, 2013).

Las estrategias políticas establecidas por la Comisión Europea (CE) y la Organización para la Cooperación y el Desarrollo Económicos (OCDE) subrayan la importancia que la bioeconomía y el cambio de la humanidad de ser dependiente de los recursos fósiles, estén ocupado un lugar importante en el espacio político y económico internacional como también el europeo. Estas estrategias han ocasionado que varios países, regiones e industrias europeas adopten y desarrollen nuevas destrezas, que expongan sus propósitos y visiones en la aplicación de una bioeconomía en Europa (De Besi \& McCormick, 2015).

Gracias a la intervención de la Comisión Europea dentro de la bioeconomía en Europa se incluyó la formación del panel europeo de bioeconomía, que tiene como principal objetivo afrontar las contradicciones dentro del margen político de la Unión Europea. El panel de bioeconomía busca integrar las políticas a nivel nacional y regional, proponiendo instaurar paneles de bioeconomía a nivel interdepartamentales y regionales para extender la colaboración y coherencia (De Besi \& McCormick, 2015). La integración está sustentada en 3 pilares claves: 1) financiación en investigación, innovación y destrezas, 2) reforzamiento de la interacción política y el convenio de las 
partes interesadas y 3) el progreso de los mercados y la competencia en sectores bioeconómicos (De Besi \& McCormick, 2015).

A pesar de que la mayoría de los países europeos, se enfocan en desarrollar estrategias de investigación e innovación incluyen a la bioeconomía dentro de ellas ampliando estrategias de crecimiento verde o azul e incorporando estrategias orientadas en la economía circular. (Fund et al., 2015), por ejemplo, una de las políticas que cambió la Unión Europea está relacionada a los biocombustibles, ellos decidieron apoyar a los biocombustibles de segunda generación los cuales son elaborados a partir de biomasa lignocelulósica, es decir, que estos biocombustibles se forman a partir de los residuos de sembríos o de cultivos en tierras marginales (Lewandowski, 2015).

\section{Países de África con políticas y estrategias de Bioeconomía explicitas.}

En el Continente Africano encontramos a 10 países (Kenia, Mali, Mauricio, Mozambique, Namibia, Nigeria, Senegal, Sud-África, Tanzania y Uganda) que toman en cuenta a la bioeconomía dentro de sus políticas ya sea como estrategia o como promotora del desarrollo del país, cada uno de los países ubica su lineamiento de acuerdo a sus necesidades, como se muestra en la Tabla 3.

Uno de los grandes problemas que ha tenido el Conteniente Africano durante todo el tiempo está relacionado principalmente con la escases de alimentos; el incremento y desarrollo agrícola es primordial para el progreso económico y social de África porque esto contribuye a la generación de empleo, ingresos, seguridad alimentaria y generación de riqueza, lo que permite que más africanos obtengan la ansiada seguridad alimentaria percibiendo una mejor vida trabajando en el sector agrícola (Virchow, Beuchelt, Kuhn \& Denich, 2016).
Tabla 3

Lineamientos de los países africanos

\begin{tabular}{ll}
\hline $\begin{array}{l}\text { Orientación de la estrategia de } \\
\text { Bioeconomía }\end{array}$ & \multicolumn{1}{c}{ País } \\
\hline Biotecnología & Kenia \\
Bioenergía & Mali \\
Economía azul & Mauricio \\
Bioenergía & Mozambique \\
Investigación e Innovación & Namibia \\
Bioenergía & Nigeria \\
Bioenergía & Senegal \\
Desarrollo holístico de la bioeconomía & Sud-África \\
Biotecnología & Tanzania \\
Bioenergía, Biotecnología & Uganda \\
\hline
\end{tabular}

Fuente: (Fund et al., 2015).

La progresiva demanda de productos fabricados a base de biomasa convertirá a la agricultura africana de ser el sector que distribuye alimentos a un proveedor de biomasa (Bracco, Calicioglu., Gómez \& Flammini, 2018) como también convertirse en un sector estratégico de procesamiento en la creciente bioeconomía internacional (Poku, Birner, Gupta, 2018a), estos productos incluirían bienes agrícolas no alimentarios, energía y materias primas industriales, que darán como resultado a una economía basada en la biomasa, que no solo produciría recursos biológicos renovables sino que además convertiría la biomasa en productos para diferentes usos (Bracco et al., 2018). Esto se convertiría en una gran oportunidad para muchos países africanos y sus habitantes, más que un peligro para su estabilidad alimentaria y nutricional, estimulando y extendiendo el desarrollo económico gracias a la dotación de recursos naturales y capital humano (Virchow et al., 2016).

La revolución de la bioeconomía en África 
incrementará los vínculos entre la fabricación, la transformación y la comercialización de la biomasa (Bracco et al., 2018). Por ejemplo países como Ghana, Malawi, Tanzania y Etiopía están empezando a ser parte de diferentes labores y actividades bioeconómicos relacionadas con los diferentes cultivos de materias primas (Poku et al., 2018a).

La aplicación del concepto de bioeconomía según la OCDE, 2008 significa transformar el "conocimiento de las ciencias de la vida en productos nuevos, sostenibles, ecoeficientes y competitivos". Para África sería un atributo particular, pero de igual manera un enorme desafío.

Los países africanos de escasos ingresos, pero abundantes en biomasa tienen la gran facilidad de no solo compensar su propia demanda futura de materias primas, sino que además pueden suministrar biomasa a países de mayores ingresos y muy necesitados de biomasa (crudo y procesados). Un ejemplo claro se encuentra en los países europeos que para desarrollar su estrategia de bioeconomía, dependen de las importaciones de biomasa. (Virchow et al., 2016). Al existir una demanda en aumento de alimentos y diferentes materias primas a base de biomasa, como cultivos de combustibles y fibras, la bioeconomía emergente de África tendría un importante espacio para desarrollar políticas estratégicas en todo el continente (Poku, Birner, Gupta, 2018b).

No obstante, a estas ventajas, en el continente africano, solamente la República de Sudáfrica ha delineado una estrategia de bioeconomía, mientras que fuera del continente muchos países en desarrollo la han aplicado (Virchow et al., 2016). La estrategia de bioeconomía de Sudáfrica se concentra en la bioinnovación y la salud de la agricultura, la industria y el medio ambiente, pero actualmente aún no se han perfeccionado mediciones para examinar el rendimiento, pero si han identificado los sectores económicos estratégicos que son: agricultura, salud, industria y medio ambiente (Bracco et al., 2018).

La estrategia bioeconómica de la República sudafricana está basada en 4 objetivos principales: 1) fortalecer la competitividad del país internacionalmente con mayor énfasis en el sector industrial y agrícola, 2) instaurar empleos más sostenibles, 3) optimizar la seguridad alimentaria y 4) fundar una economía más verde a medida que el país se direcciona hacia una economía baja en carbono (Bracco et al., 2018).

Esa estrategia africana tiene diferentes indicadores para inspeccionar el avance de la bioeconomía en relación con otros países de ingresos altos y medios, considerablemente divididos en indicadores de: apoyo financiero (gasto interno bruto en I + D como porcentaje del PIB y financiamiento y apoyo gubernamental) y de conocimiento y habilidades (investigadores equivalentes a tiempo completo, publicaciones científicas y publicaciones relacionadas con la bioeconomía) (Bracco et al., 2018)

La bioeconomía en África incrementará las relaciones entre la fabricación y el procesamiento de la biomasa, así como también proporcionará a los países de menores ganancias y sometidos a la agricultura a crear empleos e ingresos en el sector de la producción, procesamiento y mercadeo de la biomasa, en plazas urbanas y rurales. Los desafíos clave son identificar caminos para que los países pobres y los productores pobres aprovechen estas oportunidades, preguntas como ¿qué tipos de biomasa?, ¿Qué 
tipo de procesamiento y tecnologías?, ofrecen una oportunidad realista para los productores y procesadores de biomasa en estos países (Virchow et al., 2016).

\section{Países de Asia con políticas y estrategias de Bioeconomía explicitas.}

En muchos países del mundo el concepto de bioeconomía se ha implementado para el desarrollo de políticas y estrategias, pero en Asia no muchos países aplican este concepto, (D'Hondt, Jiménez-Sánchez \& Philp, 2015) a pesar de que la energía a base de biomasa tiene una fuerte relación con la bioeconomía que es utilizada en diferentes países asiáticos (Raychaudhuri \& Kumar, 2016). En Asia 9 países (China, India, Japón, Indonesia, Malasia, Rusia, Corea del Sur, Sri Lanka y Tailandia) han documentado y puesto a circular información acerca de la Bioeconomía y la definición explicita de políticas o estrategias, y además han establecido lineamientos para su desarrollo, como se muestra en la Tabla 4 (German Bioeconomy Council, 2015).

La bioeconomía es un referente crítico en el continente asiático, por ejemplo, en el sudeste del continente el término de bioeconomía es muy diferentes a otras economías desarrolladas con respecto a la biomasa. Existe una gran suma de biodiversidad en algunos países del sudeste asiático en relación a otros países pertenecientes a la OCDE. (D'Hondt et al., 2015). Muchas de las economías nacientes asiáticas están catalogadas entre las naciones más innovadoras del planeta. No debe de sorprendernos encontrar estrategias políticas de bioeconomía que estén basadas en el desarrollo de la alta tecnología, las industrias emergentes y la innovación industrial como sucede en Malasia, Corea del Sur, India, Japón y China. En el caso de Tailandia y Sri Lanka
Tabla 4

Lineamientos de los países asiáticos

\begin{tabular}{ll}
\hline Orientación de la estrategia de Bioeconomía & \multicolumn{1}{c}{ País } \\
\hline Biotecnología, Investigación e Innovación & China \\
Biotecnología & India \\
Investigación e innovación, Economía & \\
circular & Japón \\
y Desarrollo regional & \\
Bioenergía & \\
Economía de base biológica & Indonesia \\
Investigación e Innovación & \\
Desarrollo holístico de la Biotecnología & Malasia \\
Biotecnología & Rusia \\
Biotecnología & Corea del Sur \\
Biotecnología & Sri Lanka \\
Biotecnología & Tailandia \\
Bioenergía & \\
\hline
\end{tabular}

Fuente: (Dieckhoff et al., 2015; Fund et al., 2015)

atraídos por esas estrategias han desarrollado su bioeconomía principalmente en las áreas de biotecnología y la bioindustria. Indonesia ha centrado sus estrategias bioeconómicas en las cadenas de valor del sector agrícola, pero con un enfoque a gestionar el suministro de energía y alimentos en el archipiélago (Fund et al., 2015).

De los 45 países que han documentado y puesto a circular información acerca de la Bioeconomía y la definición explicita de políticas o estrategias, y además han establecido lineamientos para su desarrollo (Fund et al., 2015), algunos países asiáticos se han limitado a seguir este propósito, siendo uno de estos Malasia con su plan de bioeconomía (Bioeconomy Malaysia 2014) es un ejemplo considerable que ha desarrollado un plan estratégico ambicioso (D'Hondt et al., 2015), en Malasia el aceite de palma es el recurso primordial para el desarrollo socioeconómico y bioeconómico del país, 
(Sadhukhan et al., 2018), en el 2014 generó ganancias de 12 mil billones de dólares estadounidenses en exportaciones, lo que convirtió al país en pionero y líder de la industria en concepto de capital (Pye, 2018).

Actualmente los cultivos de la industria del aceite de palma están situados en zonas industriales abandonadas y en reservas de baja emisión de carbono, Igualmente, Malasia tiene una política en relación al biodiesel en donde demanda que los fabricantes de combustible combinen el aceite de palma y el diésel derivado del petróleo con el diésel que se utiliza para el transporte (Sadhukhan et al., 2018).

Otro país interesado en la bioeconomía es India, para ellos la bioeconomía es una economía sostenible que comprende actuaciones biotecnológicas en los sectores alimenticios, energías, recuperación de recursos, diagnósticos, atenciones sanitarias y el cuidado del medio ambiente, empleando recursos renovables para la elaboración de productos biológicos, con base en el desarrollo holístico, la bioeconomía India tiene una capacidad de incrementarse a más de $\$ 100$ mil millones para el 2025 con una tasa de crecimiento anual del 20\% (Venkata, Chiranjeevi, Shikha \& Naresh, 2018).

Aunque Japón no cuenta con un plan estratégico de bioeconomía, la considera en sus políticas actuales para construir una (D'Hondt et al., 2015). Países como China, India, Japón, Corea y Taiwán son los mejores postulantes para evolucionar en el desarrollo de la biotecnología para comenzar el cambio a una nueva bioeconomía al momento que la dependencia económica del petróleo este decreciendo en el futuro (Lee, 2016).

En el continente asiático la bioeconomía resulta enormemente atractivas gracias a su expansión territorial y posición geográfica; cuenta con una gran cantidad de biomasa y una significativa importación de petróleo (D'Hondt et al., 2015), algunas economías situadas en el sudeste asiático ya cuentan con un incremento bioeconómico de dos dígitos (Sheppard Gillespie, Hirsch, Begley, 2011). Aunque el cambio climático afecta mucho a Asia y las políticas de moderación del cambio climático son muy costosas, la bioeconomía podría ofrecerle grandes aportes y oportunidades económicas, ambientales y sociales (D'Hondt et al., 2015). Por Ejemplo, India rápidamente se convertirá en el mercado más extenso para productos genéticamente modificados (GM), además será el mercado más perceptivo para la inversión en este tipo de productos en toda Asia, la industria de los bioplásticos será dominada por China e India y por otro lado Japón estará inmerso en financiar e invertir la producción de bioindustrias de alta tecnología (Lee, 2016).

Los países asiáticos a largo plazo se convertirían en líderes en producción de biomasa y gracias a la investigación y desarrollo promulgarían una bioeconomía a gran escala. (D'Hondt et al., 2015) Asia se encuentra preparada al contar con el mayor número de jóvenes biotecnólogos de todo el planeta, pero también cuentan con el mayor número de habitantes con el índice de desarroIlo más bajo, si Asia junto con su extenso número de recursos biológicos explotaran las bioindustrias o bioempresas se podrían suprimir estos grandes contrastes de desigualdad (Dey, 2014).

\section{Países de Oceanía con políticas y estrate- gias de Bioeconomía explicitas.}

A pesar de que Oceanía cuenta con 14 países únicamente Australia y Nueva Zelanda tie- 
nen establecidos sus políticas y lineamientos en relación a la bioeconomía, como se muestra en la Tabla 5 (German Bioeconomy Council, 2015). Estos países han concentrado sus esfuerzos en crecimiento y elaboración de valor a las industrias primarias. (Staffas, Gustavsson \& McCormick, 2013).

Uno de los grandes desafíos que enfrenta Australia es la escasez de agua, el impacto climático en su ambiente frágil y el incremento de la energía renovable (Staffas et al., 2013), aunque Australia no cuenta con una estrategia oficial de bioeconomía, el gobierno ha direccionado su participación política y sustento en distintos sectores temáticos de la bioeconomía, concretamente en la afinidad al incremento del valor agregado de los recursos agrícolas, forestales y marinos (Fund, et al., 2015). Australia tiene un gran atributo comparativo en concepto de biorrefinería, porque sus dos factores importantes como el clima y el sector agrícola garantizan un enorme abastecimiento de material de biomasa y es usado específicamente en la elaboración de productos plásticos, químicos y combustibles (O'Hara \& Glenn, 2017).

Las políticas correspondidas con la bioeconomía australiana pueden determinarse como

Tabla 5

\section{Lineamientos de los países australianos.}

\begin{tabular}{ll}
\hline \multicolumn{1}{c}{ Orientación de la estrategia de } & País \\
\hline \multicolumn{1}{c}{ Bioeconomía } & \\
Investigación e Innovación & Australia \\
Economía azul & \\
Desarrollo Regional de Bioeconomía & \\
Bioenergía & Nueva \\
Economía de base biológica & Zelanda \\
Investigación e Innovación & \\
\hline
\end{tabular}

Fuente: (Dieckhoff et al., 2015; Fund et al., 2015) estrategias de I+D. En el año 2013, el gobierno australiano definió 15 prioridades estratégicas de también constituyen temas clave de la bioeconomía como por ejemplo la bioenergía, el monitoreo y gestión de ecosistemas, alimentosy salud optimizados (Fund, et al., 2015).

El gobierno australiano ha presentado varias estrategias separadas en diferentes documentos para las áreas específicas y en una de esas áreas toma a la bioeconomía para asuntos especiales. El documento define a la bioeconomía como un concepto emergente, que no está aun sólidamente establecido, del cual la agrobiotecnología es un elemento clave, es decir la estrategia se centra mayormente en la biotecnología relacionándola con la bioeconomía mas no con la economía de base biológica.

La Organización de Investigación Científica e Industrial del Commonwealth (CSIRO), que es la agencia nacional de ciencia en Australia, creó una página web relacionada a la bioeconomía en la cual da a conocer las líneas de investigación australiana que ocupan de áreas como la bioseguridad y la adaptación al clima, la agricultura sostenible y las cuestiones relacionadas con el agua, las mismas que están relacionadas directamente con la bioeconomía. Así Mismo el CSIRO se ha dedicado a publicar artículos científicos sobre la bioseguridad en una bioeconomía ascendente, en donde se afrontan los peligros del uso de materias primas modificadas biológicamente principalmente para la elaboración de alimentos y otros fines. Este es uno de los temas que tienen gran relación con la bioeconomía, pero podemos decir que en la práctica Australia está ampliamente interesado en el desarrollo de una economía de base biológica, pero que se encuentra distribuidas en diferentes documentos estratégicos (Staffas et al., 2013).

Al igual que Australia, Nueva Zelanda no cuen-

Pensamiento Americano Vol. 11 (22) · 2018 • Julio-Diciembre · Corporación Universitaria Americana • Barranquilla, Colombia • ISSN: $2027-2448$. 
ta con una estrategia específica de bioeconomía, en el 2012 el país toma primordialmente la utilización de la economía de base biológica como una estrategia de competitividad llamada "Agenda de Crecimiento Empresarial". En este sentido Nueva Zelanda formó una estrategia de innovación específicamente para el sector primario y biológico que en el 2013 fue lanzado como "Fondo de Investigación de Industrias Biológicas" (Fund et al., 2015).

El Gobierno de Nueva Zelanda ha explorado el potencial de transformación del sector biotecnológico en donde ha activado recursos mediante agencias nacionales y regionales, como también de organizaciones investigativas para apresurar la elaboración de una bioeconomía, además reconocen el potencial de transformación del sector biotecnológico, por tal motivo han movilizado recursos a través de agencias nacionales y regionales, así como de organismos de investigación para acelerar la construcción de una bioeconomía progresista (Ahn, Meeks, Bednarek, Ross \& Dalziel, 2010).

\section{Referencias}

Aguilar, A., Wohlgemuth, R. \& Twardowski, T. (2018). Perspectives on bioeconomy, New Biotechnology, 40, 181-184.

Ahn, M., Meeks, M., Bednarek, R., Ross, C. \& Dalziel, S. (2010). Towards a high-performance bioeconomy: Determining cluster priorities and capabilities in New Zealand. International Journal of Commerce and Management, 20(4), 308-330.

Álvarez, C., Amorós, J. E. \& Urbano, D. (2014). Regulations and Entrepreneurship: Evidence from Developed and Developing Countries. Innovar, 24 (spe), 81-89. https://dx.doi.org/10.15446/innovar.v24n1spe.47548

Asada, R. \& Stern, T. (2018). Competitive Bioeconomy? Comparing Bio-based and Non-bio-based Primary Sectors of the World, Ecological Economics, 149, $120-128$.

Biber-Freudenberger, L., Kumar Basukala, A., Bruckner, M. \& Börner, J. (2018). Sustainability Performance of National Bio-Economies. Sustainability, 10(2705), 1-20.

Biotalent Canada. (2013). Sequencing the Data - People - Driving Canada's Bio-economy, Labour Market Information Report 2013. Recuperado de: https:// www.biotalent.ca/sites/biotalent/files/PDF/Sequencing/mobile/index.html

Bracco, S., Calicioglu, O. \& Gómez, M. \& Flammini, A. (2018). Assessing the Contribution of Bioeconomy to the Total Economy: A Review of National Frameworks. Sustainability, 10 (6), 1-17.

D’Hondt, K., Jiménez-Sánchez, G. \& Philp, J. (2015). Reconciling Food and Industrial Needs for an Asian Bioeconomy: The Enabling Power of Genomics and Biotechnology. Asian Biotechnology and Development Review, 17(2), 85-130.

De Besi, M. \& McCormick, K. (2015). Towards a Bioeco-

Pensamiento Americano Vol. 11 (22) • 2018 • Julio-Diciembre • Corporación Universitaria Americana • Barranquilla, Colombia • ISSN: $2027-2448$. 
nomy in Europe: National, Regional and Industrial Strategies. Sustainability, 7, 10461-10478.

Dey, S. (2014). Asian bioeconomy and biobusiness: current scenario and future prospects. New Biotechnology, 31, s34.

Dieckhoff P., El-Cichakli B. \& Patermann C. (2015). Bioeconomy Policy. Synopsis and Analysis of Strategies in the G7. A Report from the German Bioeconomy Council. Office of the Bioeconomy Council: Berlin, Germany.

Duchesne, L. \& Wetzel, S. (2003). The bioeconomy and the forestry sector: Changing markets and new opportunities. The Forestry Chronicle, 79(5). 860864

Dupont-Inglis, J. \& Borg, A. (2018). Destination bioeconomy - The path towards a smarter, more sustainable future. New Biotechnology, 40 (PART A), 140-143.

Fund, C., El-Chichakli, B. \& Dieckhoff, P. (2015). Bioeconomy Policy (Part II). Synopsis of National Strategies around the World. A Report from the German Bioeconomy Council. Office of the Bioeconomy Council: Berlin, Germany.

Golden, J. S., Handfield, R. B., Daystar, J. \& McConnell, T. E. (2015). An Economic Impact Analysis of the U.S. Biobased Products Industry: A Report to the Congress of the United States of America. United States Department of Agriculture.USA. Recuperado de: https://www.biopreferred.gov/BPResources/files/ EconomicReport_6_12_2015.pdf

Goven, J. \& Pavone, V. (2015). The Bioeconomy as Political Project: A Polanyian Analysis. Science, Technology, \& Human Values, 40(3), 302-337. https://doi. org/10.1177/0162243914552133

Henry, G., Pahun, J. \& Trigo, E. (2014). La Bioeconomía en América Latina: oportunidades de desarrollo e implicaciones de política e investigación. FACES, 20(42-4), 125-141.
Imbert, E., Ladu, L., Morone, P. \& Quitzow, R. (2017). Comparing policy strategies for a transition to a bioeconomy in Europe: The case of Italy and Germany. Energy Research \& Social Science, 33, 70-81.

Lee, D. (2016). Bio-based economies in Asia: Economic analysis of development of bio-based industry in China, India, Japan, Corea, Malaysia and Taiwan. International Journal of Hydrogen Energy, 41(7), 43334346.

Lewandowski, I. (2015) Securing a sustainable biomass supply in a growing bioeconomy. Global Food Security $6,34-42$.

Lokko, I., Heijde, M., Schebesta, K., Scholtès, P., Van Montagu, M. \& Giacca, M. (2018). Biotechnology and the bioeconomy-Towards inclusive and sustainable industrial development, New Biotechnology, 40, 5-10. DOI: 10.1016/j.nbt.2017.06.005

McCormick, K. \& Kautto, N. (2013). The Bioeconomy in Europe: An Overview. Sustainability, 5, 2589-2608.

Meyer, R. (2017). Bioeconomy Strategies: Contexts, Visions, Guiding Implementation Principles and Resulting Debates. Sustainability 9(1031), 1-32.

Mustalahti, I. (2017). The responsive bioeconomy: The need for inclusion of citizens and environmental capability in the forest based bioeconomy. Journal of Cleaner Production, 172, 3781-3790.

O'Hara, I. \& Glenn, D. (2017). The Economic Case For Bioeconomy Development In Australia. Industrial Biotechnology, 13(2), 65-68.

Patermann, C. \& Aguilar, A. (2018). The origins of the bioeconomy in the European Union. New Biotechnology, 40, 20-24.

Philp, J. (2018) The bioeconomy, the challenge of the century for policy makers. New Biotechnology, 40, 11-19. DOI: 10.1016/j.nbt.2017.04.004 
Poku, A., Birner, R. \& Gupta, S. (2018a). Is Africa ready to develop a competitive bioeconomy? The case of the cassava value web in Ghana. Journal of Cleaner Production, 200, 134-147.

Poku, A., Birner, R. \& Gupta, S. (2018b). Making Contract Farming Arrangements Work in Africa's Bioeconomy: Evidence from Cassava Outgrower Schemes in Ghana. Sustainability, 10(5), 1-21.

Pye, O. (2018). Commodifying sustainability: Development, nature and politics in the palm oil industry. World Development, 1-11.

Raychaudhuri, A. \& Kumar Ghosh, S. (2016). Biomass Supply Chain in Asian and European Countries. Procedia Environmental Sciences, 35, 914-924.

Sadhukhan, J., Martínez-Hernandez, E., Murphy, R. J., Ng, D. K. S., Hassim, M. H., Siew Ng, K. \& Andiappan, V. (2018). Role of bioenergy, biorefinery and bioeconomy in sustainable development: Strategic pathways for Malaysia. Renewable and Sustainable Energy Reviews, 81(2), 1966-1987.

Sasson, A. \& Malpica, C. (2017). Bioeconomy in Latin América, New Biotechnology, 40 (2018), 40-45.

Scarlat, N., Dallemand, J. F., Monforti-Ferrario, F. \& Nita, V. (2015). The role of biomass and bioenergy in a future bioeconomy: Policies and facts. Environmental Development, 15, 3-34.

Sheppard, A., Gillespie, I., Hirsch, M. \& Begley, C. (2011). Biosecurity and sustainability within the growing global bioeconomy. Current Opinion in Environmental Sustainability, 3(1-2), 4-10.

Sillanpää, M. \& Ncibi, C. (2017). Bioeconomy: Multidimensional Impacts and Challenges. In: A Sustainable Bioeconomy. Springer, Berlin: Cham, 317-343.

Staffas, L., Gustavsson, M. \& McCormick, K. (2013). Strategies and Policies for the Bioeconomy and Bio-Based Economy: An Analysis of Official National
Approaches. Sustainability, 5(6), 2751-2769.

Trigo, E., Henry, G., Sanders, J., Schurr, U., Ingelbrecht, I., Revel, C., Santana, C. \& Rocha, P. (2013). Towards bioeconomy development in Latin América and the Caribbean. Bioeconomy Working Paper (1), 1-15.

Vega, M. \& Madrigal, O. (2017). International Bioeconomy Innovations in Central America. En Dabbert, S., Lewandowski, l., Weiss, J. \& Pyka, A. (Eds). Knowledge-Driven Developments in the Bioeconomy, Economic Complexity and Evolution, (83-96). Springer, Cham.

Venkata Mohan, S., Chiranjeevi, P., Shikha Dahiy, A. \& Naresh Kumar, A. (2018). Waste derived bioeconomy in India: A perspective. New Biotechnology, 40, PART A, 60-69.

Virchow, D., Beuchelt, T.D., Kuhn, A. \& Denich, M. (2016). Biomass-Based Value Webs: A Novel Perspective for Emerging Bioeconomies in Sub-Saharan Africa. In: Gatzweiler F. \& von Braun, J. (Eds) Technological and Institutional Innovations for Marginalized Smallholders in Agricultural Development, 225-238. Switzerland. Springer, Cham.

German Bioeconomy Council (2015), Bioeconomy Policy (Part II) - Synopsis of National Strategies around the World. Recuperado de: https://biooekonomierat.de/en/
2018, Vol. 11(22) 230-246 (C)The Author(s) 2018 Reprints and permission: www.americana.edu.co https://www.coruniamericana.edu.co/publicaciones/ojs/index.php/pensamientoamericano/index 